infections of rectal GC and CT were found giving an incidence rate of 91.2 per 100 person years. The incidence rate was highest when consecutive visits were within 30 days $(241.8$ per 100 person years, HR 0.83, $\mathrm{p}=0.00$ ). There were no significant associations of incidence of rectal gonorrhoea and chlamydia with demographic and behavioural characteristics (Abstract O1-S10.05 table 1).

Conclusions A high prevalence of asymptomatic rectal gonorhoea and chlamydia was observed along with a high incidence rate of these infections among high-risk MSM in India. The persistence of high prevalence of asymptomatic infections remaining even after presumptive treatment at the first visit calls for a review of the periodicity of presumptive treatment in similar clusters of MSM. Consistent condom use and partner treatment need to be reemphasised.

\section{1-S10.06 HIV/STI PREVALENCE AMONG MEN WHO HAVE SEX WITH MEN IN 4 CITIES, CHINA AND ASSOCIATED RISK FACTORS FOR HIV INFECTION}

doi:10.1136/sextrans-2011-050109.60

X Chen, N Jiang, B Wang. National Center for STD Control, China CDC, Nanjing, China

Objectives The data on STIs control and HIV prevention is limited among MSM population. To examine STIs control strategies for HIV prevention in a community-based cohort of HIV-negative MSM, community intervention trail was conducted between 2009 and 2011. This report mainly discussed baseline survey results.

Methods This study was conducted in MSM community of four mid-sized cities from Jul. to Sep. 2009. All participants were recruited through venue-based recruitment, complemented by peer referral using snowball method, Questionnaire were completed in STD clinics or VCT centers. Blood samples were collected for HIV, syphilis and HSV-2 tests, and urine sample for CT/NG PCR tests. Results $35.3 \%$ participants self-identified as homosexual and $44.7 \%$ bisexual. The most popular way to seek male sexual partners was internet (38.4\%). $15.9 \%$ of participants had provided or acquired sex services with male, while $19.1 \%$ of respondents reported unprotected anal intercourse (UAI) in the last sex services. 3.8\% of participants had experienced sadism \& masochism(SM). In past 6 months, $80.8 \%$ of participants had anal sex with man and $29.0 \%$ reported UAI during the last intercourse. $38.5 \%$ of participants reported having had sex with woman and only $45.2 \%$ of those reported using condom during the last intercourse with woman. $18.0 \%$ of participants involved in commercial sex services had taken drugs such as methamphetamine, Ketamine and MDMA.10.6\% of participants was HIV infection. $34.4 \%$ of participants is TP-ELISA positive results indicated a history of syphilis infection, and $20.9 \%$ were both positive results of ELISA and TRUST indicated active syphilis. $3.0 \%$ of participants were tested as NG infection, $6.8 \%$ was CT infected, and $16.2 \%$ were HSV-2 infected. Significant factors associated with HIV infection were self-reported STD infection history $[\mathrm{AOR}=2.1,95 \% \mathrm{CI}$ : $1.29 \%$ to $4.26 \%]$, syphilis infection $[\mathrm{AOR}=2.70,95 \% \mathrm{CI}: 1.81 \%$ to $4.04 \%]$, and HSV-2 infection [AOR $=3.07,95 \% \mathrm{CI}: 2.09 \%$ to $4.50 \%$ ].

Conclusions MSM have been potential bridge-population for HIV/ STIs from most-at-risk population to general population. Intervention activities should target the internet, sexual social networks, and certain subpopulations such as those taking drugs in commercial sex services or infected with STIs. Friendly and high-quality STIs service should reach to MSM who do not attend STD clinics. Campaigns are urgent not only to boost individual condom use but also to create culture for condom use in MSM community.

\section{Epidemiology oral session 11: Various topics} of special interest

\section{1-S11.01 TIME EVOLUTION OF THE FRACTION OF NEW HIV INFECTIONS DUE TO PRIMARY INFECTION AMONG HIGH RISK GROUPS IN SOUTHERN INDIA}

doi:10.1136/sextrans-2011-050109.6

${ }^{1} \mathrm{M}$ Pickles, ${ }^{1} \mathrm{M} \mathrm{C}$ Boily, ${ }^{2} \mathrm{P}$ Vickerman, ${ }^{3} \mathrm{~B} \mathrm{M}$ Ramesh, ${ }^{4} \mathrm{R}$ Washington, ${ }^{5} \mathrm{~K}$ Deering, ${ }^{3} \mathrm{~S}$ Verma, ${ }^{6} \mathrm{~J}$ Blanchard, ${ }^{6} \mathrm{~S}$ Moses, ${ }^{7} \mathrm{M}$ Alary. ${ }^{1}$ Imperial College London, UK; ${ }^{2}$ LSHTM, UK; ${ }^{3}$ Karnataka Health Promotion Trust, Bangalore, India; ${ }^{4}$ St John's Research Institute, Bangalore India; ${ }^{5}$ University of British Columbia, Canada; ${ }^{6}$ University of Manitoba, Winnipeg, Canada; ${ }^{7}$ Centre hospitalier affilié universitaire de Québec, Quebec, Canada

Background HIV infectivity is considerably higher during primary infection (PI: first few months after infection). PI is believed to be an important biological driver of HIV transmission at the population level, which has implication for prevention. We aimed to estimate the contribution of PI to HIV spread among high-risk groups in southern India.

Methods We used incidence predictions from a bespoke dynamical model of HIV transmission among FSW/clients parameterised and fitted to district-specific behavioural data and HIV/STI prevalence estimates, within a Bayesian framework. Multiple rounds of crosssectional survey data from among FSW/clients of Mysore and Belgaum, carried out as part of the evaluation of Avahan, the India AIDS initiative, were used to inform plausible parameter ranges and estimate HIV prevalence. Assumed risk of HIV infection (RRHIV/ PI) during PI was elevated by 4.5- to 19-fold compared to asymptomatic infections (based on systematic review) and lasted for 3 to 6 months. The annual fraction of new HIV infections (AF) due to primary infection was estimated over time from the 196 and 796 posterior parameter sets fitting Mysore and Belgaum data, respectively.

Results The median AF (overall: clients+FSW) declined from $57.3 \%$ ( $\min =34, \max =82$ ) \% in 1987 (assumed start of epidemic) to $49 \%$ $(29,70) \%$ in $2000,43.7 \%(25,65) \%$ in $2004,23.8 \% / 24.3 \%(10 / 10,42 /$ $43) \%$ in 2010/2025 for Mysore (Abstract O1-S11.01 figure 1). Similar AF estimates were obtained in Belgaum and for FSWs and clients separately in both districts. The univariate association between AF and RRHIV/PI declined slightly over time (Abstract O1-S11.01 figure 1). In multivariate regression analysis, RRHIV/PI (slope $=0.027$ ), duration of the whole infectiousness period (slope $=-0.014$ ) and duration of primary infection (slope $=0.587$ ) were significantly associated with AF early in the epidemic (1988) $(\mathrm{R} 2=0.833$, $\mathrm{p}$ value $<0.001)$. However, the AF in 2025 was also associated with number of years selling sex by street-based FSW (slope $=-0.020, p$ value $=0.042$ ) and number of years buying sex by high-activity clients (slope $=-0.004, p$ value $=0.055)(R 2=0.684)$.

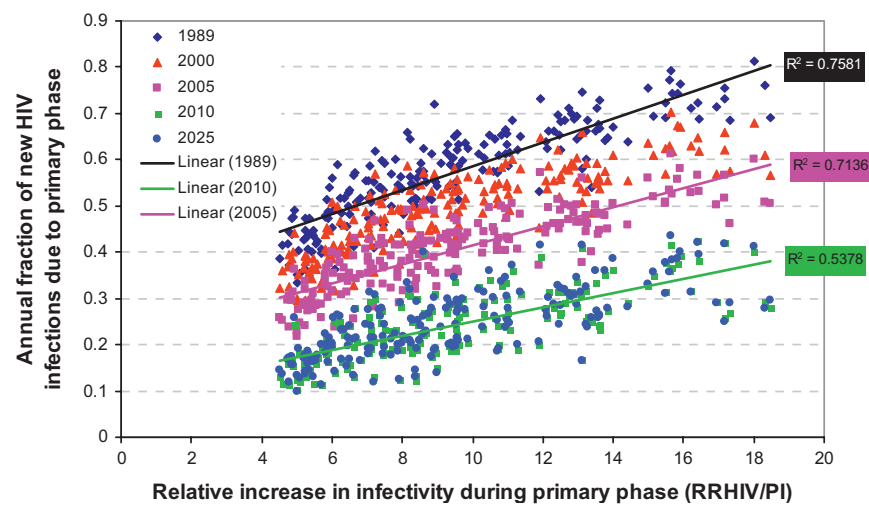

Abstract 01-S11.01 Figure 1 FSW and clients combined Mysore. 
Conclusions The role of primary infection decreased as the HIV epidemic matured but could still account for a large fraction of new infections, especially if RRHIV/PI is above 10. Early on, its contribution depended on parameters of primary infections. Later on, its contribution also depended on the renewal of high-risk susceptible population, which fuels HIV incidence.

\section{1-S11.02 DETERMINANTS OF TIME TRENDS IN HIV PREVALENCE IN THE YOUNG ANTENATAL POPULATION OF KARNATAKA DISTRICTS}

doi:10.1136/sextrans-2011-050109.62

${ }^{1} \mathrm{U}$ Mahajan, ${ }^{2} \mathrm{P}$ Banandur, ${ }^{3} \mathrm{~S}$ Rajaram, ${ }^{3} \mathrm{~T}$ Duchesne, ${ }^{3} \mathrm{~B}$ Abdous, ${ }^{4} \mathrm{~B} \mathrm{M}$ Ramesh, ${ }^{5} \mathrm{~S}$ Moses, ${ }^{6} \mathrm{M}$ Alary. ${ }^{1}$ CHARME /I project, Bangalore, India; ${ }^{2}$ CHARME II project, 2 Rajarajeswari Medical College and Hospital, Bangalore, India; ${ }^{3} \mathrm{CHARME}$ II project, Bangalore, India; ${ }^{4}$ Karnataka Health Promotion Trust, Bangalore, India; ${ }^{5}$ University of Manitoba, Winnipeg, Canada; ${ }^{6}$ Centre hospitalier affilié universitaire de Québec, Quebec, Canada

Background In 2003, the Bill \& Melinda Gates Foundation initiated a focused HIV prevention program (India AIDS Initiative: Avahan) among high-risk and bridge groups. We studied determinants of time trends in HIV prevalence among young ( $<25$ years) antenatal (ANC) women caused by these intensive prevention intervention (IPI) program compared to non-intensive intervention (Non-IPI) program.

Methods Random intercept multilevel models were developed using logistic regression (xtmelogit command) to examine effects of IPI, program and district level variables on HIV prevalence among young ANC women. Data from annual sentinel surveillance of ANC women were used as individual level characteristics. Selected program and socio-demographic variables at district level were included as distal variables. Interaction between time and intensity of program intervention was assessed.

Results HIV prevalence in young ANC women decreased steadily from $1.4 \%$ to $0.77 \%$ from 2003 to 2007 , and increased to $0.83 \%$ in 2008 (Adjusted OR (AOR) $=0.59$, (95\% CI): $0.45 \%$ to $0.77 \%$ ). Rural ( $\mathrm{AOR}=0.87,95 \% \mathrm{CI}: 0.76 \%$ to $0.99 \%$ ) and literate women ( $\mathrm{AOR}=0.76,95 \% \mathrm{CI}: 0.66 \%$ to $0.87 \%$ ) had lower risk of HIV compared to urban and illiterate women respectively. Presence of major truck halt points ( $\mathrm{AOR}=1.57,95 \% \mathrm{CI}$ : $1.17 \%$ to $2.12 \%$ ) in the district was associated with high risk of HIV. Higher age at marriage was associated with lower risk of HIV ( $\mathrm{AOR}=0.85,95 \% \mathrm{CI}$ : $0.78 \%$ to $0.93 \%)$. There was significant interaction between time and intensity of intervention. In the years 2006 and 2007, Non-IPI districts had a significantly higher risk of HIV compared to IPI districts (AOR2006 $=1.86$, 95\% CI: $1.18 \%$ to $2.93 \%$ and AOR 2007 $=2.25$, $95 \%$ CI: $1.39 \%$ to $3.62 \%$ ) respectively. Among the program variables regular contact with high risk group was associated with slightly reduced risk of $\mathrm{HIV}(\mathrm{AOR}=0.998,95 \% \mathrm{CI}$ : $0.996 \%$ to $0.999 \%$ ) see Abstract O1-S11.02 table 1.

Conclusion HIV prevalence in ANC population declined significantly in IPI districts from 2003 to 2008 compared to non-IPI districts in Karnataka. IPI had a definite impact on reduction of HIV prevalence in general population during 2006 and 2007. This coincides with the maturity of IPI during 2006 and 2007 along with the initiation of NACP-III in 2007. Learning from IPI might have influenced National AIDS Control Program-III implementation in non-IPI districts in Karnataka leading to similar effects in IPI and non-IPI districts in 2008. Improving female literacy and increasing the age at marriage would help favour reduction of HIV.
Abstract 01-S11.02 Table 1 Determinants of time trends in HIV prevalence in the young antenatal population of Karnataka districts

\begin{tabular}{|c|c|c|}
\hline $\begin{array}{l}\text { Individual/district level } \\
\text { characteristics }\end{array}$ & $\begin{array}{l}\text { Null model } \\
\text { AOR }(95 \% \mathrm{CI})\end{array}$ & $\begin{array}{l}\text { Random intercept model: } \\
\% \text { high risk group persons } \\
\text { contacted regularly } \\
\text { AOR }(95 \% \mathrm{CI})\end{array}$ \\
\hline \multicolumn{3}{|l|}{ Fixed part of the model } \\
\hline Constant & $\begin{array}{l}0.009(0.0080 \text { to } \\
0.0114)\end{array}$ & 0.528 (0.097 to 2.8782$)$ \\
\hline \multicolumn{3}{|l|}{ Individual characteristics } \\
\hline \multicolumn{3}{|l|}{ Year-2003 (Reference) } \\
\hline 2004 & & $0.992(0.7854$ to 1.2521$)$ \\
\hline 2005 & & $0.804(0.6287$ to 1.0288$)$ \\
\hline 2006 & & $0.585(0.4458$ to 0.7681$)$ \\
\hline 2007 & & $0.418(0.3109$ to 0.562$)$ \\
\hline 2008 & & $0.585(0.4475$ to 0.7649$)$ \\
\hline \multicolumn{3}{|l|}{ Locality-Urban (Reference) } \\
\hline Rural & & $0.867(0.7581$ to 0.9915$)$ \\
\hline \multicolumn{3}{|l|}{ Type of site-District headquarter (Reference) } \\
\hline First referral unit-rural & & $0.784(0.6904$ to 0.8907$)$ \\
\hline \multicolumn{3}{|l|}{ Literacy-Illiterate (reference) } \\
\hline Literate & & $0.759(0.6622$ to 0.871$)$ \\
\hline \multicolumn{3}{|l|}{ Type of intervention-IPI (reference) } \\
\hline Non-IPI & & $0.871(0.5578$ to 1.361$)$ \\
\hline \multicolumn{3}{|l|}{ Programme variable } \\
\hline$\%$ Contacted regularly & & $0.998(0.9959$ to 0.9999$)$ \\
\hline \multicolumn{3}{|l|}{ District level variables } \\
\hline Mean age at marriage for girls (years) & & $0.848(0.7773$ to 0.9261$)$ \\
\hline Major truck halt points & & $1.573(1.166$ to 2.1212$)$ \\
\hline \multicolumn{3}{|l|}{ Interaction } \\
\hline Year 2004 and Non-IPI & & $1.101(0.7184$ to 1.6887$)$ \\
\hline Year 2005 and Non-IPI & & $1.443(0.929$ to 2.2427$)$ \\
\hline Year 2006 and Non-IPI & & $1.856(1.1769$ to 2.9253$)$ \\
\hline Year 2007 and Non-IPI & & $2.245(1.3902$ to 3.6249$)$ \\
\hline Year 2008 and Non-IPI & & $1.098(0.6477$ to 1.8601$)$ \\
\hline \multicolumn{3}{|l|}{ Random part of the model } \\
\hline District-level variance: total & $\begin{array}{l}0.1333 \text { (0.0642 to } \\
0.2767)\end{array}$ & $0.072(0.0337$ to 0.1530$)$ \\
\hline
\end{tabular}

\section{1-S11.03 HOW MANY INFECTIONS ARE AVERTED BY BEHAVIOUR CHANGE AFTER EARLY HIV DIAGNOSIS \& COUNSELLING OF MSM? ESTIMATES FROM A STOCHASTIC INDIVIDUAL-BASED MODEL}

doi:10.1136/sextrans-2011-050109.63

${ }^{1} \mathrm{P}$ White, ${ }^{2} \mathrm{~J}$ Fox, ${ }^{1} \mathrm{~N}$ MacDonald, ${ }^{3} \mathrm{~J}$ Weber, ${ }^{3} \mathrm{M}$ McClure, ${ }^{3} \mathrm{~S}$ Fidler, ${ }^{1} \mathrm{H}$ Ward. ${ }^{1} / \mathrm{mperial}$ College, School of Public Health, London, UK; ${ }^{2}$ Guys \& St Thomas' NHS Trust / Kings

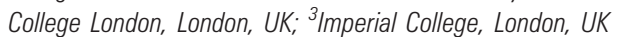

Background A recent paper (Fox et al HIV Medicine 2009) reported that MSM in the UK significantly reduced their transmission-risk behaviour following HIV diagnosis and suggested that this could be effective in averting transmission during the highly-infectious primary infection stage. However, cost-effectiveness analysis is required to inform policy-making. To assess the effectiveness of early HIV diagnosis in MSM as a prevention strategy we quantified its potential impact in terms of transmission HIV events averted.

Methods We developed an individual-based stochastic transmission model to calculate the number of HIV-transmission events expected to occur from a cohort of recently-diagnosed MSM with and without the changes in behaviour that occurred post-diagnosis and counselling. The model incorporates different types of sex-act, patterns of condom use, and distinguishes between regular and casual partners. 